\title{
L'UNIFICAZIONE DEL DIRITTO PENALE EUROPEO CON RIFERIMENTO ALLA TURCHIA (*)
}

\author{
Doç. Dr. Yılmaz GƯNAL
}

Senza dubbio lo sviluppo storico dell'opinione sulla unificazione dell'Europa ha un passato lontano. Lunghi secoli di storia, filosofi, scrittori, uomini diplomatioi e di Chiesa, studiosi, si sono occupati di questo progetto, dimostrando la volontà ed il desiderio di metterlo in pratica. Fermarsi su ciô, perô, non porterà ad al. cun risultato pratico in merito all'argomento che tratterô mentrevoglio qui solo ricordare la proposta fatta, a suo tempo, dall'avvocato francese PIERRE DUBOIS di una confederazione dei Paesi Cristiani contro i turchi invasori. Questa si puô dire essere stata la prima volta in oui l'Europa abbia avuto una iniziativa di unificazione, iniziativa ripetuta dopo il 1453 dal re di Boemia GEORGES PODIEBRAD, anche questa volta contro l'impero ottomano. Ho riportato questo per dire che, essendo turco, sono contento che le vicende storiche del mio popolo siano servite, se non altro, almeno a lanciare la prima idea di una Europa unita.

Mentre questa prima idea di unificazione era nata solo per uno scopo difensivo, nel periodo intercorrente le due guerre mondiali tale scopo ha cambiato le sue caratteristiche. Cosi è nata la Paneurope, il Consiglio Economico di Paneurope, collaborazione economicodoganale dell'Europa, il progetto di BRIAND, della collaborazione economico-politico dell'Europa.

Dopo la seconda guerra mondiale l'idea dell'unificazione dell'Europa ha avuto un ulteriore sviluppo, assumendo un aspet-

(*) Ringrazio sentitamente il Proffesore BETTIOL per avermidata la opportunità di paticipare con questa mia comunicazione anche al quarto Convegno penalistico internazionale di BRESSANONE. 
to piû realistico. Le prime iniziative sono venute dalla Inghilterra con il movimento della Europa Unita e idella Francia. Le assocciazioni create dopo la seconda guerra mondiale possono avere due scopi comuni, l'uno, l'unificaziona dell'Europa dal punto di vista politicio ed economico e, l'altro di difesa.

La Turchia non ha potuto astenersi dall'aderire a tutti i movimenti di unificazione anche se, essendo politicamente un paese europeo, geograficamente sia un cuscinetto euro-asiatico. La Turchia infatti si sente molto piû vicina all'Europa che alla Asia poichè per noi l'Europa è uno scopo, un incentivo per progredire. La Turchia è presente come membro nella maggioranza delle assocciazioni euro. pee come: O.E.C.E.; U.E.P.; A.M.E.; N.A.T.O.; C.E.M.T.; E.U.R.O.F.I. M.A.; C.E.A.C.; C.E.P.T. Ci sono altre assocciazioni secondarie create per l'unificazione europea, e alle quali la Turchia ha partecipato. Possiamo citare alcune di queste: B.R.I. (1930), C.E.P.P. (1950), C.I.E.C. (1950) ecc.

Possiamo quindi osservando questi dati vedere come la Turchia non ha potuto sentirsi estranea alla integrazione Europea e che anzi ha cercato di collaborare per essa, perciô possiamo dire anticipamente che siamo d'accordo per la unificazione di un diritto penale europeo.

Vogliamo brevemente soffermarci su qualche movimento positivo dell'unificazione nel campo sopra detto e che a noi maggiormente interessa, quello del diritto penale ed in speciale modo possiamo citare i lavori del Consiglio Europeo. I risultati scaturiti da questi movimenti, appertengono piuttosto alla parte generale del diritto penale, come la misura di prevenzione dei reati, e della criminalità e collaborazione reciproca per la punizione dei rei.

Dopo avere fatto qualche accenno possiamo chiederci: possiamo unificare il diritto penale? Possiamo arrivare ad una codificazione europea? Quali sono le basi essenziali per arrivare a ciô e quali possibilità di successo puô avere l'opinione di una unificazione del diritto penale europeo?

Senza dubbio non è possibile con questa breve discussione cercare le risposte a domande di questo genere, per lo meno dettagliamente, accontentiamoci quindi di discutere e appianare l'argomento almeno a grosse linee. 
Entro subito nel vivo dell'argomento ricordando solo che le disertazioni che ne farô non mirano ad essere delle teorie ma sono il riflettersi delle soluzioni o interpretazioni prospettate dalla Turchia stessa secondo la propria situazione giuridica, sociale economica odierna. Dopo avere quindi studiato il problema basandomi su materiale di una ricerca da me compiuta posso dire di essere l'alfiere delle idee e delle prospettive sulla Turchia sulle soluzioni all'argomento da noi trattato e mi sembra giusto, che ogni singolo Stato costruisca prima il pezzetto di mosaico che poi porterà a contributo del grande quadro che è la unificazione europea.

A mio modesto avviso i quesiti che direttamente sorgono dalla trattazione di questo argomento sono tre :

1) che cosa si puô unificare in un futuro diritto penale europeo

2) come si puô arrivare ad una unificazione del diritto penale europeo

3) accettando come reale e già avvenuta l'unione europea penale esaminare quali siano i problemi derivanti dalla applicazione di un futuro codice europeo.

Inizio rispondendo alla prima domanda dicendo che è possibile, anzi utile, discutere sull'unificazione del diritto penale iniziando dalla sua parte generale. Non credo infatti ci sia molta utilità pratica avere delle differenze nei principi generali poichè l'esperienza insegna che nella parte generale del diritto penale $i$ vari codici europei non hanno in fondo grandi e inconciliabili differenze.

Se, dopo una ricerca approfondita, si potrà arrivare ad avere dei principi generali di diritto penale comuni ai Paesi europei non ci saranno opposizioni scientifiche o giuridiche alla applicazione di detti principi e, se vi saranno, non saranno logiche. Senza dubbio sorgeranno dei contrasti, per esempio tra Paesi Occidentali o Orientali, o tra sistemi concernenti le varie misure di sicurezza o nel trattare la individualizzazione della pena ma non credo che tali opposizioni possano essere insolubili.

Non credo di potere invece difendere tanto facilmente come sopra l'unificazione della parte speciale del diritto penale poichè, essa parte, è lo specchio della società e vi è strettamente legata piû di ogni altro ordinamento giuridico. I reati, le prevenzioni genera- 
li e speciali, i mezzi di punizione dipendono dai fattori etico socialimorali-economici di ciascun popolo. In questo campo si presenta infatti una impossibilità pratica da parte di tutti i differenti Paesi di giungere ad uno stadio comune dei suddetti valori.

Dicendo questo teniamo conto anche dei codici speciali di ogni Paese che debbono essere unificati. Si pensi che solo la Turchia ne ha quasi mille.

In quanto alle contravvenzioni per la unificazione penale di esse si presentano le stesse difficoltà che possono presentarsi dalla unificazione dei reati. Per me le contravvenzioni pur essendo un illecito penale sono prevenzioni a reati piû gravi, perciô, considerandole sempre un illecito penale, non ci sarà un contrasto tra il rifiutare la unificazione delle contravvenzioni e il non accettare quelle della parte speciale.

Dal punto di vista dogmatico è possibile essere di accordo anche sui principi generali della parte speciale del diritto penale. E' possibile esemplificare ed appianare le formalità che attualmente regolano la metaria giuridica nei vari paesi europei come è possibile attuare una collaborazione piû stretta per la prevenzione dei reati e per la lotta contro la criminalità.

Si puô arrivare ad un accordo anche sul livello piû alto nel trattamento dei condannati e nella espiazione della pena, tenendo perô presente che il livello al quale si penserà di arrivare non dovrà essere solo quello attuato nei paesi socialmente piû avanzati poichè non potrà essere superiore, e di molto, a quello in cui la maggioranza dei condannati era abituato da libero. Questo almeno allo stato attuale delle disparità sociali tra i singoli Paesi, disparità che si colmeranno solo con il tempo. Per quanto riguarda l'espiazione della pena si dovrà accettare si che l'essere privati della libertà possa essere una sanzione di per sè sufficiente ma non dimentichiamo che il reo ha pur sempre commesso un reato e che quindi deve sentire che sta espiando una pena.

Sono del parere che anche per le istituzioni post-penitenziarie si possa giungere ad una unificazione, arrivando, forse lo dico un poco ottimisticamente, ad uno scambio di ex condannati fra i diversi Paesi, onde tenerli un poco lontani dal loro solito ambiente.

A prima vista questa mia idea puô sembrare alquanto campata in aria $\mathrm{o}$, per lo meno, estremista ma, tenendo sempre presente che 
la pena ha principalmente lo scopo di rieducare e di riadattare nella società il condannato, puô felicemente inserirsi nel quadro delle future istituzioni post penitenziarie internazionali.

Importante sarebbe anche raggiungere un accordo sul sistema di detenzione per i condannati di sesso femminile e per i minorenni, sistemi che solo in pochi Paesi attualmente sono soddisfacenti.

Passo ora ad esaminare il secondo punto propostomi: a mio avviso i mezzi di unificazione possono essere tre:

a) adottare, anzi tradurre un codice penale scelto e pensato come il migliore attualmente in vigore e prenderlo per modello.

Per dimostrare come questo sistema sia da ritenersi per lo meno insufficiente, apro una parentesi che credo interessante. Fino dal 1926 in Turchia è in vigore il codice di Zanardelli, codice da noi adottato per estrema necessità dovendo cambiare tutto l'ordinamento giuridico islamico fino ad allora in vigore. Secondo me questo codice come principi generali contiene delle istituzioni di alto valore ma nella applicazione da noi fatta non posso nascondere che ne sono scaturite molte difficoltà. Non credo che allo stato delle cose attuali ci sia una necessità incalzante di dovere adottare letteralmente un codice anche perfetto.

Si potrà quindi arrivare ad una seconda strada e cioè :

b) preparare dogmaticamente un codice teoricamente perfetto valido per tutti i Paesi europei. Anche se questo codice sarà preparato dai piû insigni legislatori senza alcun fondamento etico sociale morale economico di un livello comune europeo non potrà adattarsi ai singoli caratteri societari. Anche a questo proposito posso citare l'esperienza recentemente fatta dalla Turchia, esperienza che potrà essere riflessa sull'unità europea qualora si decida di attuare un tale sistema di codificazione.

Come punto di partenza ho preso il 1960 perchè oltre ad essere la data di una rivoluizone politica, anche i lavori di modificazione del codice penale hanno preso maggiore intensità.

Con il codice costituzionale del 1961 qualche istituzione 
del diritto penale ha ottenuto una garanzia costituzionale, ad esempio: il divieto di tortura, divieto di dare una pena inconciliabile con la dignità umana, segreto della vita privata, inviolabilità di domicilio, libertà di corrispondenza, di idee e di culto, libertà di pensiero, della stampa, dí riunione, libertà di appellarsi al diritto; legalità e personalità della pena, l'obbligo della esistenza delle condizioni necessarie a causare l'arresto, libertà di prova, immunità parlamentare, imparzialità dei Tribunali, garanzia per i giudici; i Tribunali superiori e quello Costituzionale hanno preso luogo dettagliatamente nel nostro nuovo Codice Costituzionale.

D'altra parte l'ordinamento del diritto penitenziario ha avuto un grande cambiamento con le modifiche apportate dal Proff. LOPEZ REY con il codice numero 647. Abbiamo scritto diversi articoli su queste moldifiche che non si possono attuare praticamente in un breve periodo di preparazione. Infatti per attuare delle modifiche in un campo cosi importnante quale è quello del diritto, bisogna conoscere molto bene la struttura di quella determinata società, studiarla a fondo, conoscerne le abitudini e l'indole, in speciale modo quando il popolo al quale si apporteranno le modifiche é adirittura straniero al legislatore. Malgrado tutto il Codice è entrato in vigore. Lo scopo principale del nuovo codice era quello di risparmiare economicamente tramutando l'esecuzione della pena in un breve periodo. Forse questo era giusto, infatti secondo il calcolo del 1963 nelle carceri turche avevamo 172.764 persone ed il $40^{\circ} \%^{\circ}$ di loro erano in arresto. II $78^{\circ} \%$ dei rimanenti scontavano una pena inferiore ai 6 mesi. Mentre prima le spese per le carceri erano di 25.000 .000 di Lire Turche, con il nuovo sistema si poteva fare un risparmio del $62^{\circ} /{ }^{\circ}$ cioè 17.500 .000 Lire Turche erano pronnte per la riforma penitenziaria. Questi calcoli erano giusti solo teoricamente, ossia sulla carta, ma d'altra parte erano conformi alle istruzioni date dalle Nazioni Unite e dal Consiglio Europeo.

Il nuovo codice inoltre sostituisce certi provvedimenti alla detenzione prevista per le condanne fino ai 6 mesi, per i delitti stradali colposi e per i delitti colposi prevede solo 
una pena pecuniaria, I limiti della sospensione condizionale della pena sono allargati. E prevista la esecuzione della pena nel domicilio, o solo quale fine settimana, libertà condizionata, istituzioni di centri di osservazione dei condannati sono alcune modifiche apportate dal codice numero 647. Immediatamente dopo la messa in atto delle nuove norme sono sonti grandi contrasti. Un esempio: il prevedere una pena pecuniaria per i reati colposi derivanti da incidenti stradali hanno aumentato gli incidenti montali cosi che la Turchia, pur avendo la minore intensità dei veicoli circolanti, ha raggiunto il primo posto come incidenti mortali, tanto che secondo le statistiche del O.E.C.D. e E.C.M.T. abbiamo avuto un aumento di incidenti del $45^{\circ} \%$.

Per la Turchia quindi il codice attualmente in vigore puô essere ottimo e conforme ad un certo livello eurepeo ma non possiamo e dobbiamo trascurare le difficoltà e gli inconvenienti che risultano dalla sua applicazione.

Provvedere, per esempio, ai condannati un permesso di cercare un lavoro senza avere l'istituzione del patronaggio non servirà a niente, o prevedere per i condannati minorenni un diverso tipo di espiazione della pena senza neanche avere le carceri sufficenti per loro e con la prospettiva idi attendere da 20 anni l'istruzione di un tribunale per i minorenni.

Ho trovato interessante riferire quesito per dire che una utopia codicistica penale avrebbe forti ripercussioni in campo sia nazionale che internazionale.

Per necessità di fatto quindi dobbiamo cercare e formulare una terza strada per arrivare all'unificazione di un codice penale.

c) Anche nel campo del diritto penale possiamo applicare il criterio moderno secondo cui un lavoro di equipe è necessario ad attuare una ricerca. Creare quindi in seno di ogni singolo Paese questa direi squadra non solo di penalisti, logicamente, infatti il penalista deve itenere conto dell'apporto dello statista, sociologo, criminologo psicologo di massa e dell'individuo, che collaborando fra loro studi- 
no a fondo, su basi reali e probanti, come sia stato applicato il Codice nazionale, le conseguenze della sua applicazione, che cosa si possa aggigungere e che cosa togliere; solo dopo questa preparazione potranno riunirsi, le varie equipe nazionali e, collaborando fra loro, potranno trovare dei punti in comune. Solo cosi si pottrà essere sicuri che le nuove istituzioni internazionaliche veranno decise, siano consone anche alle varie nazioni, poichè si avrà sempre tenuto conto, probando scientificamente, dello ambiente nazionale e delle necessità di ogni singolo Paese. Questa è la parte veramente dinamica del problema.

Ammettendo a questo punto che si possa arrivare ad una unificazione nel campo del diritto penale europeo, come immediata conseguenza sorgerà un altro problema e non indifferente: quello della applicazione del diritto penale europeo e passaggio cioè di quello statico a quello dinamico. E logico che questo diritto, e che io mi permetto di chiamare dinamico, abbia in se tutte le garanzie a un livello europeo di libertà per e accusato. Anche questo perô, facendo un passo avanti non basterà piû perchè avere un codice statico-dinamico perfetto senza avere dei giudici con una preparazione, cultura, umanità comune fra di loro non basterà ad affrontare le inevitabili conseguenze che nasceranno da un diritto penale europeo.

Basti pensare alla individualizzazzione della pena che secondo mè sorge dalla coscienza dei giudici.

Inoltre quando immaginiamo un diritto penale unificato necessariamente dobbiamo pensarlo investito da una garanzia sopra costituzionale.

Molto si discute sulla possibilità di un diritto penale unificato, molto si critica, molto si spera, ma, pur coscienti delle difficoltà che logicamente nascono dal problema dobbiamo constatare che è lampante come l'Europa cerchi una unificazione in vari settori, settori che richiederanno necessariamente l'urgente formazione di un diritto unificato che ordini le conseguenze derivanti dalle alleanze. Come esempio: basti pensare che c'è un M.E.C., c'è quindi uno scambio economico in seno ai vari Stati, ma ben poco fino 
ad ora si è fatto per un diritto penale ecenomico. Per una ricerca da me fatta posso ben idire che i reati internazionali economici che aspettano una soluzione valida sono migliaia. Mi rendo conto che allo stato attuale delle cose si voglia, e si tenti di fare un diritto penale unificato solo nello ambito della piccola Europa, ma a mio avviso, bisogna essere coraggiosi e guardare sin dall'inizio ad una Europa piû vasta, sia per trarre giovamento dalle esperienze altrui, sia per mitigare e superare quel desiderio di supremazia che giustamente i singoli Stati costituenti la piccola Europa vantano nel campo del diritto.

Per concludere dirô che, io credo nella necessità di un diritto penale europeo perchè credo profondamente nello uomo, che ha saputo elevarsi dalla vita primitiva fino agli spazi e che quindi vincerà tutte le difficoltà nascenti dal problema propostoci. L'unico vero punto oscuro secondo mè, è sapere che l'uomo è quasi sempre di carattere debole o si lascia facilmente prendere dall'orgoglio e da antiche prevenzioni. 